\title{
Experimentelle Untersuchungen zur radialen Durchschallung von Stämmen mittels variabler Frequenz
}

\author{
Sebastian Clauss • Fritz Bächle $\cdot$ Peter Niemz
}

Published online: 16 Dezember 2006

(C) Springer-Verlag 2006

\begin{abstract}
Zusammenfassung Es wurden unterschiedliche Kenngrössen von Ultraschallsignalen für die Erkennung von Defekten in Stämmen getestet. Es zeigte sich, dass durch Messung der Schallgeschwindigkeit die besten Ergebnisse erzielt werden. Proben mit einer künstlich eingebrachten Bohrung mit $30 \mathrm{~mm}$ Durchmesser konnten über die Schallgeschwindigkeit von solchen mit $100 \mathrm{~mm}$ Durchmesser separiert werden. Das Füllen der Hohlräume mit Sägespänen bewirkte keinen Unterschied der Messergebnisse. Die Parameter RMS, Peak to Peak, Amplitude und Signalenergie erbrachten keine verwertbaren Ergebnisse. Die Signalamplitude korreliert stark mit der Dichte und der Holzfeuchte.
\end{abstract}

\section{Radial scanning of logs \\ by sound using variable frequencies}

Abstract Different characteristic values
nals were used for evaluating pieces of
that sound velocity attains best results for
and defects in the used samples. Samp
and with holes of $30 \mathrm{~mm}$ diameter could
rated from samples with holes of $100 \mathrm{~mm}$
it did not matter if the holes were empty
S. Clauss
Institut für Holz- und Papiertechnik,
Lehrstuhl für Holz- und Faserwerkstofftechnik,
TU Dresden,
Dresden, Germany
F. Bächle · P. Niemz ( $)$
Institut für Baustoffe, Holzphysik, ETH Zürich,
HIF E 25.2, Schafmattstr. 6,
8093 Zürich, Switzerland
e-mail: niemzp@ethz.ch

Abstract Different characteristic values of ultrasound signals were used for evaluating pieces of log. It was shown that sound velocity attains best results for describing holes and defects in the used samples. Samples without holes and with holes of $30 \mathrm{~mm}$ diameter could be clearly separated from samples with holes of $100 \mathrm{~mm}$ diameter. Thereby it did not matter if the holes were empty or filled with

S. Clauss

Institut für Holz- und Papiertechnik,

TU Dresden,

Dresden, Germany

F. Bächle · P. Niemz (

HIF E 25.2, Schafmattstr. 6,

e-mail: niemzp@ethz.ch strands or sanding dust. It seems that the sound runs faster along the intact wood and thus going round the hole and not through the filled hole. The attenuation of the signal can be used as a further parameter for describing the samples. Both, sound velocity and attenuation are frequency dependent. Otherwise RMS, Peak to Peak and signal energy provided no information for distinguishing the samples. The amplitude of the signal is strongly dependent on moisture, density and connection to the wood. These parameters cannot be separated easily from the search values. So far, in practical applications only sound velocity can be used reliably for evaluating trees and describing flaws in trunks.

\section{Einleitung}

Die Durchschallung von Holz in radialer Richtung, aber auch die Schalltomographie sind gebräuchliche Methoden zum Nachweis von Fäule, Rissen oder anderen inneren Defekten in Bäumen. Eingesetzt werden dazu Geräte mit unterschiedlicher Frequenz und verschiedenem Funktionsprinzip (Wellenerzeugung durch Quarz oder durch mechanisches Anschlagen). Die Frequenz schwankt dabei zwischen $300 \mathrm{~Hz}$ und $100 \mathrm{kHz}$. Ausgewertet wird meist die Laufzeit, teilweise auch die Energie der Signale (z.B. beim Sylvatest 2). Für die Laufzeitmessung mit konstanter Messfrequenz sind verschiedene Messgeräte (z.B. Sylvatest, Fakopp, PICUS) verwendbar.

Im Rahmen der Arbeit wurde mit einem vom FraunhoferInstitut für Zerstörungsfreie Prüfverfahren Saarbrücken, Außenstelle EADQ Dresden, speziell für diese Messungen für Holz entwickelten Gerätesystem gearbeitet. Um die Reproduzierbarkeit zu erhöhen, erfolgten die Messungen an Stammabschnitten aus Fichtenholz mit künstlich einge- 
brachten Defekten. Dabei wurden die Messfrequenz und der Auswertealgorithmus variiert.

\section{Erkenntnisstand}

Nachfolgend wird eine Übersicht zu wesentlichen Einflussfaktoren auf die Schallgeschwindigkeit gegeben.

Rohdichte und Elastizitätsmodul: Die Schallgeschwindigkeit in Holz wird durch das Verhältnis E-Modul zu Dichte bestimmt. Je größer dieses Verhältnis ist, desto größer ist auch die Schallgeschwindigkeit.

Holzfeuchte und Temperatur: Die elastomechanischen Eigenschaften des Holzes werden wesentlich durch die Holzfeuchte und die Temperatur beeinflusst. Mit zunehmender Holzfeuchte als auch mit steigender Temperatur sinkt der E-Modul. Dieser Einfluss wirkt sich analog auf die Schallgeschwindigkeit aus. Die Schallgeschwindigkeit nimmt mit zunehmender Holzfeuchte und Temperatur ab.

Holzstruktur: Strukturelle Einflüsse, wie die Jahrringbreite, der Faserwinkel sowie der Winkel zwischen radialer und tangentialer Richtung, die den E-Modul beeinflussen, wirken sich ebenso auf die Schallgeschwindigkeit aus. Bei Messungen parallel zur Faserrichtung ergeben sich höhere Schallgeschwindigkeiten als senkrecht dazu. In radialer Richtung ergeben sich höhere Schallgeschwindigkeiten als in tangentialer Richtung. Ein Minimum der Schallgeschwindigkeit bildet sich bei $45^{\circ}$ aus.

Probengeometrie: Die Probengeometrie hat ebenfalls einen Einfluss auf die Schallgeschwindigkeit. Bucur (1995) ermittelte eine sinkende Schallgeschwindigkeit mit steigendem Verhältnis von Probenbreite zu Probenhöhe. Niemz und Bächle (2002) sowie Niemz et al. (2002) zeigten, dass die Schallgeschwindigkeit bei Stoßwellen $(16 \mathrm{kHz})$ mit zunehmendem Stammdurchmesser ansteigt, bei einer Frequenz von $50 \mathrm{kHz}$ dagegen abfällt. Ebenso verweisen Sandoz und Lorin (1994) auf einen Abfall der Schallgeschwindigkeit mit zunehmendem Stammdurchmesser.

Holz zerstörende Pilze: Ein Pilzbefall hat je nach Pilzart eine unterschiedliche Wirkung auf das Holz. Braunfäuleerreger befallen vor allem Nadelbäume und bauen Zellulose und Hemizellulose ab. Das Holz wird dadurch brüchig, pulvrig und es kommt zu Riss- und Spaltenbildung. Die Biege- und Schlagfestigkeit des Holzes werden drastisch gemindert. Weißfäuleerreger greifen vor allem Laubbäume, seltener Nadelbäume an. Sie bauen zuerst Lignin und Hemizellulose, später auch Zellulose ab. Das Holz wird fasrig, die Festigkeitseigenschaften ändern sich weniger drastisch als bei Braunfäuleerregern. Moderfäuleerreger befallen Laub- und Nadelbäume gleichermaßen. Sie bauen ebenfalls Zellulose, Hemizellulose und Lignin ab. Das Holz wird dadurch spröde und neigt zu Sprödbruch. Ein Pilzbefall führt zu einer Veränderung des Verhältnisses von E-Modul zu Dichte, und damit ebenso zu einer Veränderung der Schallgeschwindigkeit. Arbeiten zum Einfluss des Pilzbefalls auf die Schallgeschwindigkeit führten u.a. Schwarze und Fink (1994) durch. Dabei wurden deutliche Unterschiede in der Wirkung der verschiedenen Pilze nachgewiesen. Der Schädigungsmechanismus beeinflusst wesentlich die Schallgeschwindigkeit. So sind wesentliche Unterschiede zwischen Weiß- und Braunfäule vorhanden. Pilze wie der Brandkrustenpilz sind mittels Schall kaum nachweisbar (Schwarze und Fink 1994).

Fäuleerkennung: Da Holz zerstörende Pilze das Verhältnis von E-Modul zu Rohdichte verändern und dieses proportional zur Schallgeschwindigkeit ist, eignet sich das Durchschallungsverfahren zur Detektierung von Faulstellen im Holz. Die Änderung des o.g. Verhältnisses muss dabei allerdings so groß sein, dass die gemessene Geschwindigkeit deutlich vom Referenzwert abweicht. Im fortgeschrittenen Stadium der Fäule, das heißt bei Vorhandensein von Hohlräumen läuft die Welle um diese herum und benötigt somit eine längere Wegstrecke. Die ermittelte Schallgeschwindigkeit weicht somit von der vorausberechneten - bei direktem Weg - ab und gibt Aufschluss über die Größe des Defektes. Sandoz und Lorin (1994) sowie Bethge et al. (1996) haben dazu ausführliche Untersuchungen durchgeführt.

Erkennung von Ästen: Eine Detektierung von Ästen in Faserrichtung ist mittels Schallgeschwindigkeitsmessung nur schwer realisierbar. Niemz und Kucera (1997) führten dazu Untersuchungen durch und konnten feststellen, dass lediglich Äste, die direkt in Richtung der Schallausbreitung und nahe dem Sensor lagen, eine Beeinflussung der Messergebnisse darstellten. Dies dürfte sich auch bei der Durchschallung von Stämmen auswirken.

Tabelle 1 Schallgeschwindigkeiten in gesunden Bäumen für verschiedene Messgeräte

Table 1 Sound velocities in sound trees for different measuring devices

\begin{tabular}{lcc}
\hline Baumart & $\begin{array}{c}\text { Schallgeschwindigkeit }\left[\mathrm{ms}^{-1}\right] \\
\text { Sylvatest }\end{array}$ \\
\hline $\begin{array}{l}\text { Laubbäume } \\
\text { Ahorn }\end{array}$ & $1000-1500$ & \\
Eiche & $1200-1800$ & 1900 \\
Esche & $1000-15000$ & 1800 \\
Linde & $900-1300$ & 1700 \\
Platane & $900-1200$ & 1400 \\
Rotbuche & $1100-1500$ & 1800 \\
Rosskastanie & $800-1600$ & 1700 \\
Nadelbäume & & 1600 \\
Fichte & $900-1100$ & \\
Kiefer & $1000-1200$ & 1600 \\
Lärche & $1000-1400$ & 1500 \\
Tanne & $900-1200$ & 1500 \\
\hline
\end{tabular}


Messfrequenz: Nach Messungen von Niemz und Bächle (2002) wird ein deutlicher Einfluss der Messfrequenz auf die Schallgeschwindigkeit festgestellt. Die Schallgeschwindigkeit steigt mit zunehmender Messfrequenz deutlich an. Mit dem Messgerät Impulshammer $(300 \mathrm{~Hz})$, wurden deutlich niedrigere Werte ermittelt als mit den Messgeräten Sylvatest $(16 \mathrm{kHz})$ oder BP5 $(50 \mathrm{kHz})(\mathrm{vgl}$. hierzu Tabelle 1).

\section{Material und Methoden}

\subsection{Versuchsmaterial}

Für die Versuche wurde eine Fichte mit einem Durchmesser von rund $32 \mathrm{~cm}$ ausgewählt. Der Baum stammt aus dem Lehrwald der ETH Zürich im Revier Uetliberg und wurde in $30 \mathrm{~cm}$ lange Abschnitte aufgeteilt. Diese wurden anschlieBend in einem Klimaraum bei $20^{\circ} \mathrm{C}$ und $85 \%$ rel. Luftfeuchte in saftfrischem Zustand gelagert, um eine schnelle Trocknung zu vermeiden.

Bei allen Stammabschnitten wurde die Holzfeuchte mit einem Widerstands-Feuchtemessgerät vom Typ „Bollmann Combo 100“ bestimmt. Um eine möglichst gute Ankopplung der Sensoren an die Prüfkörper zu realisieren, wurden diese entsprechend vorbereitet. Die Rinde des Stammabschnittes wurde im Bereich der Ankopplung entfernt. Je Prüfkörper wurden vier Löcher in einem Winkel von $90^{\circ}$ zueinander am Umfang des Stammabschnittes eingebohrt. Die Tiefe der Bohrungen betrug dabei $20 \mathrm{~mm}$. Der Sender ist mit einem Gewinde versehen, sodass dieser in das Holz eingeschraubt werden kann, wodurch eine starre Ankopplung erreicht wird.

Nach Abschluss der ersten Messreihe wurde in die Stammabschnitte in Längsrichtung (Faserrichtung) eine durchgängige Bohrung mit einem Durchmesser von $30 \mathrm{~mm}$ (Abb. 1) eingebracht. Im Anschluss an die zweite Messreihe wurde die Bohrung auf einen Durchmesser von $100 \mathrm{~mm}$ erweitert.

Als mittlere Darrdichte der Bohrkerne wurden $354 \mathrm{~kg} / \mathrm{m}^{3}$ ermittelt. Die mittlere Holzfeuchte betrug 54\%.
Anschließend wurden die ausgebohrten Stammabschnitte mit Hobelspänen $\left(\rho=180 \mathrm{~kg} / \mathrm{m}^{3}\right)$ sowie Schleifstaub $(\rho=$ $320 \mathrm{~kg} / \mathrm{m}^{3}$ ) gefüllt, um die Herabsetzung der Dichte im Kern des Stammes bei einer Fäule zu simulieren. Praktisch sind nur in den wenigsten Fällen geschlossene Hohlräume vorhanden.

Folgende Proben wurden verwendet:

Messreihe 1: ohne Bohrung

Messreihe 2: zentrische Bohrung mit $30 \mathrm{~mm}$ Durchmesser Messreihe 3: zentrische Bohrung mit $100 \mathrm{~mm}$ Durchmesser

Messreihe 4: zentrische Bohrung mit $100 \mathrm{~mm}$ Durchmesser, Bohrung mit Spänen aufgefüllt (Streudichte der Hobelspäne $180 \mathrm{~kg} / \mathrm{m}^{3}$ )

Messreihe 5: zentrische Bohrung mit $100 \mathrm{~mm}$ Durchmesser, mit Schleifstaub aufgefüllt (Streudichte des Schleifstaubes $310 \mathrm{~kg} / \mathrm{m}^{3}$ )

Für die Untersuchungen des Übertragungsverhaltens dienten sechs Prüfkörper für den Zustand „ohne Bohrung“ und jeweils zwei Prüfkörper für die Zustände „mit Bohrung“ und „Hobelspan- bzw. Schleifstaubfüllung“. Für die Auswertung der Kenngrößen der Ultraschallsignale wurden zehn Prüfkörper verwendet.

\subsection{Methoden}

\subsubsection{Ultraschallmessgerät}

Für die Messungen wurde ein speziell entwickeltes Ultraschallmessgerät des Fraunhofer-Institutes für zerstörungsfreie Prüfverfahren Saarbrücken, EADQ Dresden, entwickelt (Abb. 2).

Das System besteht aus einem Compact PCI- Rechner, der für diese messtechnische Applikation um ein DAQ-Board und ein spezielles Ultraschallmodul erweitert wurde. Weitere Bestandteile sind ein Aktuator und ein Sensor, mit jeweils integriertem Leistungsverstärker. Die analoge Signalverarbeitung beinhaltet einen Tiefpassfil-

\author{
Abb. 1 Stammabschnitte \\ a mit $30 \mathrm{~mm}$ Bohrung \\ b mit $100 \mathrm{~mm}$ Bohrung und \\ Sägespanfüllung \\ Fig. 1 Wood samples \\ a with a hole of $30 \mathrm{~mm}$ diameter \\ b with a hole of $100 \mathrm{~mm}$ of \\ diameter and filled with strands
}

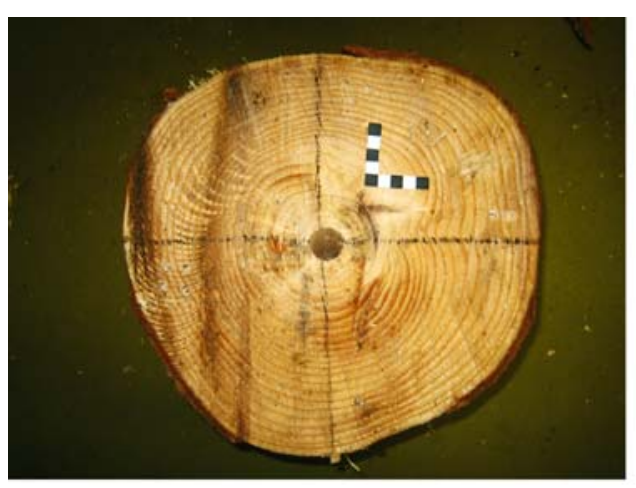

a

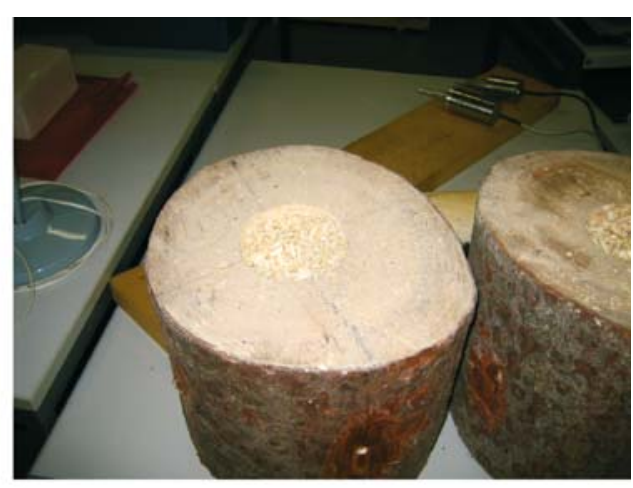

b 


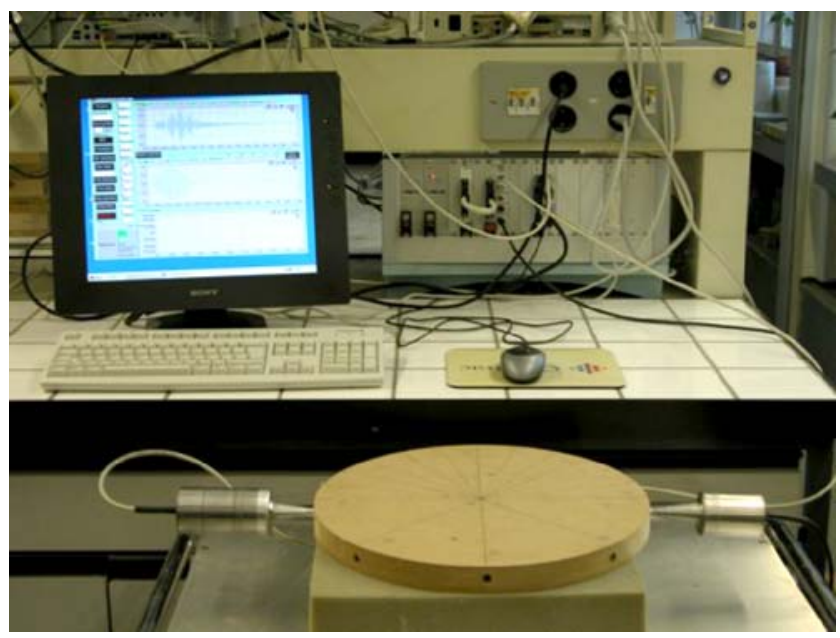

Abb. 2 Messsystem USH

Fig. 2 Measuring device USH

ter. Die $3 \mathrm{~dB}$-Grenzfrequenz liegt bei $50 \mathrm{kHz}$ und dient zur Unterdrückung von Antialiasing-Effekten. Für die Unterdrückung von niederfrequenten Modulationen wurde ein zuschaltbarer 3-stufiger Hochpass verwendet. Die Verstärkung hat den Faktor 100, die untere Grenzfrequenz liegt bei $10 \mathrm{~Hz}$, die obere bei $65 \mathrm{kHz}$. Der Aktuator besteht aus einem Metallgehäuse, welches konisch ausläuft. An dessen Ende befindet sich ein Gewinde, das der Ankopplung des Sensors an das Holz dient. Die untere Grenzfrequenz des Sensors liegt bei $300 \mathrm{~Hz}$, die obere bei $50 \mathrm{kHz}$ (Frankenstein et al. 2003).

\subsection{2 Übertragungsverhalten}

Mit Hilfe der Frequenzanalyse ist es möglich, Signale im Frequenzbereich zu untersuchen und somit Aufschluss über das Übertragungsverhalten des Signals zu erlangen. Das gesendete Signal wird dazu mit dem empfangenen Signal verglichen. Zu Beginn der Untersuchung wird ein Prüfkörper mit der Messeinrichtung USH durchschallt. Das empfangene Signal $y(k), k=1 . . N$, wird als Funktion der Zeit aufgezeichnet. Das gesendete Signal $u(k), k=1 . . N$, muss anhand der vorgegebenen Parameter mathematisch entwickelt werden, da eine Aufzeichnung nicht möglich ist. Dieser Umstand kann aufgrund der Differenzen zwischen dem eigentlichen Signal und dem Modellsignal zu nicht einschätzbaren Fehlern führen. Anschließend werden beide Signale analog Gleichungen 1 und 2 mittelwertfrei gemacht.

$y_{M}(k)=y(k)-\bar{y}(k)$,

$u_{M}(k)=u(k)-\bar{u}(k)$.

Im nächsten Schritt wird die Breite des zu betrachtenden Zeitfensters ausgewählt. Im betrachteten Fall entspricht diese der Länge des gesendeten Signals. Die Signale werden des Weiteren einer diskreten Fouriertransformation unterzogen und somit in den Frequenzbereich überführt. Innerhalb eines Fensters von 500 Samples um die Erregungsfrequenz wird anschließend für beide Signale $\hat{\Phi}_{y}^{N}$ und $\hat{\Phi}_{u}^{N}$ das Maximum der Frequenzamplitude ermittelt und in die Einheit dB umgerechnet. Diese Maximalamplituden vom empfangenen und gesendeten Signal werden ins Verhältnis zueinander gesetzt und ergeben den Übertragungsfaktor.

$\hat{G}_{N}(\omega)=\frac{\hat{\Phi}_{y}^{N}(\omega)}{\hat{\Phi}_{u}^{N}(\omega)}$.

Dieser Algorithmus wird für den Frequenzbereich von $5 \mathrm{kHz}$ bis $50 \mathrm{kHz}$ in einem Intervall von $1000 \mathrm{~Hz}$ angewendet. Dadurch ergibt sich eine Kennlinie des Übertragungsfaktors über der Frequenz. Aus dieser Kennlinie können Aussagen über das Übertragungsverhalten bei unterschiedlichen Anregungsfrequenzen getroffen werden. Sollten Defekte innerhalb des Prüfkörpers bestimmte Frequenzen stärker dämpfen, würde dies im Vergleich mit unmanipulierten Prüfkörpern ersichtlich werden.

Um bei der Frequenzanalyse einen eingeschwungenen Zustand des Prüfkörpers einzustellen, ist es notwendig, das Signal eine gewisse Zeit aufrecht zu erhalten. Diese Einschwingzeit beträgt etwa $20 \mathrm{~ms}$. Wenn bei unterschiedlichen Messfrequenzen gleich lange Signale erzeugt werden sollen, wird die Periodenzahl an die Frequenz angepasst. Somit ergeben sich Sendezeiten zwischen 12,5 ms und $50 \mathrm{~ms}$. Es wird bei allen Messungen ein Sinussignal im HanningFenster verwendet.

\subsubsection{Schallgeschwindigkeit}

Die Bestimmung der Schalllaufzeit $t_{a}$ erfolgte nach der Energiemethode. Hierbei wird der Zeitpunkt des Übergangs vom Hintergrundrauschen zum eigentlichen Signal durch einen Anstieg der Signalenergie ermittelt. Dieser Zeitpunkt gibt die Ankunftszeit des Signals an. Der Quotient aus direkter Wegstrecke d und Schalllaufzeit ergibt die Schallgeschwindigkeit v. Um beurteilen zu können, ob eine Fehlstelle im Material vorhanden ist, müssen zuerst Referenzwerte für ein fehlerfreies Objekt ermittelt werden, mit denen die Werte des Prüfobjektes verglichen werden können (Tabelle 1)

$v=\frac{d}{t_{a}}$.

Der Übergang zwischen Hintergrundrauschen und eigentlichem Signal kann durch eine Zunahme der Signalamplitude oder -energie, einer plötzlichen Änderung des Frequenzgehaltes oder irgendeiner weiteren Größe, die sich aus der aufgezeichneten Wellenform berechnen lässt, charakterisiert werden. Der am Institut für Werkstoffe im Bauwesen der Universität Stuttgart entwickelte Algorithmus zur Bestimmung der Ankunftszeit analysiert den Energieverlauf der Signale 
(Kurz et al. 2004). Die Energie eines Zeitsignals $\hat{U}\left(t_{n}\right)$ ist dabei proportional zur Summe aller quadrierten Amplituden. Als partielle Energie $E_{n}$ wird die vom Beginn der Aufzeichnung bis zum Zeitpunkt kumulierte Energie bezeichnet:

$E_{n}=\sum_{k=0}^{n}\left[\hat{U}\left(t_{k}\right)\right]^{2}$.

Abbildung 3 zeigt die Vergrößerung des Signalbeginns und den dazugehörigen Energieverlauf. Der Knick im Energieverlauf markiert deutlich den Signalbeginn. Um die Stelle dieses Knicks automatisch bestimmen zu können, wird vom Energieverlauf ein Trend subtrahiert:

$E_{n}^{\prime}=E_{n}-n \cdot \delta$.

Die Einführung eines negativen Trends ermöglicht eine einfache und flexible Bestimmung des Signalbeginns, der nunmehr als Minimum der Funktion repräsentiert ist. Der Trend ist definiert als:

$\delta=\frac{E_{N}}{N \cdot \alpha}$.

$E_{N}$ entspricht dabei der kumulierten Energie und $N$ der Anzahl aufgezeichneter Datenpunkte des gesamten Zeitsignals. Über die Wahl des Faktors $\alpha$ kann die Genauigkeit der Ankunftszeitbestimmung gesteuert werden. Ein $\alpha$ von 1 führt zu einer stabilen Bestimmung, die jedoch gegenüber dem effektiven Wert im Allgemeinen einen Zeitverzug aufweist. Mit der Erhöhung von $\alpha$ wird der Trend flacher und der Zeitverzug dadurch kleiner. Wird $\alpha$ zu groß gewählt, so wird der Trend bereits vom Rauschen überschritten, was zu einer massiven Fehlbestimmung führt. Sinnvolle Werte für $\alpha$ liegen zwischen 1 und 200.

\subsubsection{Dämpfung}

Durch Energieverlust wird die Auslenkung des gesendeten Signals vermindert. Die Dämpfung $D$ in $\mathrm{dB}$ gibt das
Verhältnis der Maximalamplitude des gesendeten zur Maximalamplitude des empfangenen Signals wieder.

$D=-20 \cdot \log \left(\frac{\max [y(k)]}{\max [u(k)]}\right)$.

\subsubsection{RMS}

Unter dem RMS (Root mean square) bzw. Effektivwert versteht man in der Elektro- bzw. Messtechnik den quadratischen Mittelwert eines periodischen Signals. Aus den aufgenommenen Daten $y(k)$ wird nach folgender Gleichung der Effektivwert berechnet.

$\mathrm{RMS}=\sqrt{\frac{1}{n} \sum_{k=0}^{n-1}[y(k)]^{2}}$

\subsubsection{Peak to Peak}

Als Peak wird die Maximalamplitude des empfangenen Signals beschrieben Der Peak to Peak-Wert gibt die Differenz zwischen maximaler positiver und negativer Amplitude des aufgenommenen Signals an.

$\mathrm{PP}=\max [y(k)]-\min [y(k)]$

\subsubsection{Signalenergie}

Die Signalenergie ist ein integrales Maß, welches durch Aufsummieren kleinster Leistungsbeträge entsteht. In der graphischen Darstellung entspricht die Signalenergie demnach der Fläche unter dem Verlauf des Zeitsignals. Bei zeitdiskreten Signalen entspricht die Signalenergie der quadratischen Norm des aufgenommenen Signals unter Ver-

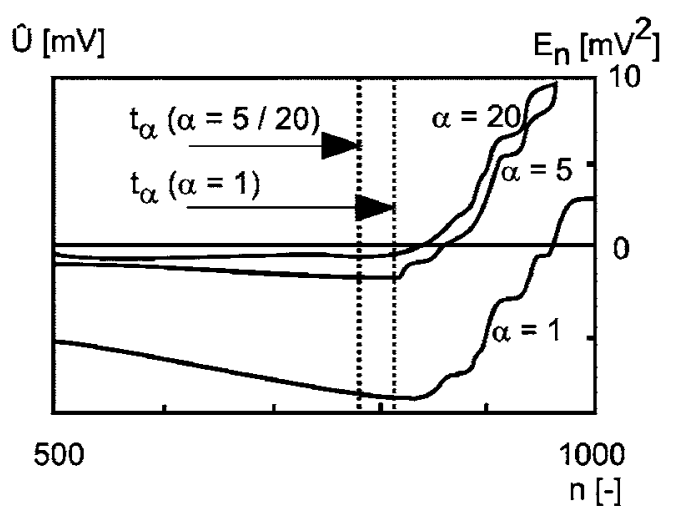

Abb. 3 Energiemethode zur Laufzeitmessung (Große und Reinhardt 1999) a Vergrößerung des Signalbeginns b Energieverlauf

Fig. 3 Energy method for runtime measuring (Große und Reinhardt 1999) a Onset of the signal (enlargement) b Behaviour of energy

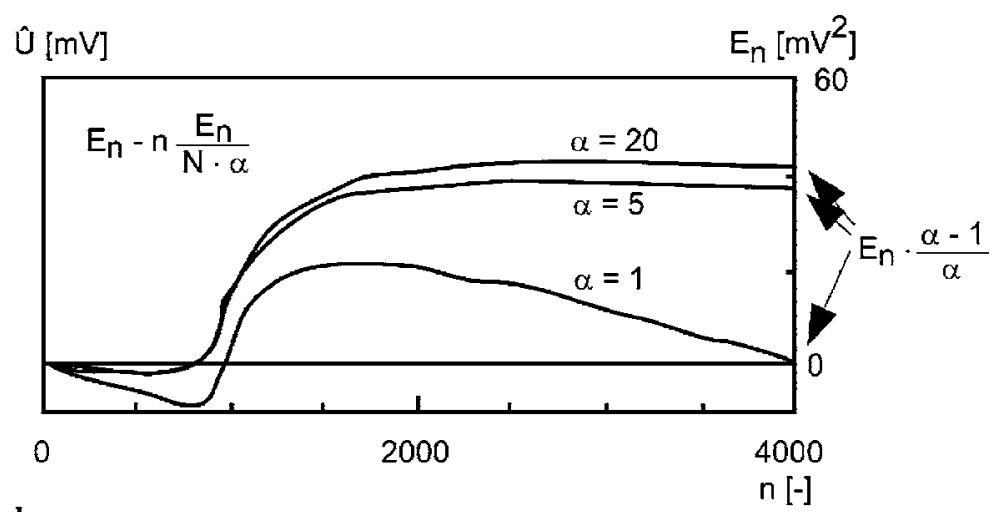

 


\section{Tabelle 2}

Einstellungsparameter zur

Kennwertberechnung

Table 2 Parameters for

evaluating the characteristic values

\begin{tabular}{lc}
\hline Signalparameter & Wert \\
\hline Samplerate & $333000 \mathrm{~Hz}$ \\
Startfrequenz & $500 \mathrm{~Hz}$ \\
Frequenzschritte & 10 \\
Endfrequenz & $50000 \mathrm{~Hz}$ \\
Periodenanzahl & 11 \\
Signalamplitude & 4 \\
\hline
\end{tabular}

wendung der Abtastperiodendauer $\Delta t_{S}$.

$E=\sum_{k=0}^{n}[y(k)]^{2} \cdot \Delta t_{S}$

\subsection{Durchführung der Messungen}

Die Messungen erfolgten an Stammabschnitten von Fichtenholz in radialer Richtung, wobei pro Stammabschnitt insgesamt vier Messungen vorgenommen wurden (Hin- und Rückmessung jeweils über Kreuz im Winkel von 90 $)$. Die Messungen zum Übertragungsverhalten fanden im Frequenzbereich von $500 \mathrm{~Hz}$ bis $50 \mathrm{kHz}$ in Schritten von jeweils $5,5 \mathrm{kHz}$ statt. Zur Kennwertberechnung wurde der gleiche Frequenzbereich mit zehn Messschritten gewählt. Tabelle 2 zeigt die Parameter der verwendeten Wellenform zur Kennwertberechnung. Über den gesamten Frequenzbereich wurde mit einem Sinussignal im Hanning-Fenster mit 11 Zyklen gemessen.

\section{Versuchsergebnisse}

\section{1 Übertragungsverhalten}

Die ermittelten Kennlinien des Übertragungsverhaltens zeigen bei allen Messreihen einen ähnlichen Verlauf über der Frequenz (Abb. 4). Die tiefen Frequenzen von $1 \mathrm{kHz}$ bis $5 \mathrm{kHz}$ sollen nicht in die Betrachtung einbezogen werden, da in diesem Bereich analoge Frequenzfilter auf das Ergebnis eingewirkt und die Messungen beeinträchtigt haben. Bei $12 \mathrm{kHz}$ zeigt sich ein erstes deutlich ausgeprägtes Maximum der Kennlinien. Im weiteren Verlauf zeigt sich bei $16 \mathrm{kHz}$ ein weiteres lokales Maximum. Bei $22 \mathrm{kHz}$ stellt sich bei allen Messreihen ein Minimum ein, wobei der Übertragungsfaktor bei der Messreihe ohne Bohrung größer ist als bei den Messreihen mit Bohrung. Die größten Übertragungsfaktoren lassen sich bei allen Messreihen in einem Frequenzbereich von $25 \mathrm{kHz}$ bis $32 \mathrm{kHz}$ feststellen. Diese Frequenzen werden demnach bei allen Messreihen am besten übertragen. Bei Messreihe 1 ergibt sich bei der Frequenz $30 \mathrm{kHz}$ nahezu keine Dämpfung der Erregungsfrequenz. Bei $30 \mathrm{kHz}$ lassen sich die Prüfkörper mit den unterschiedlichen Eigenschaften gut differenzie-

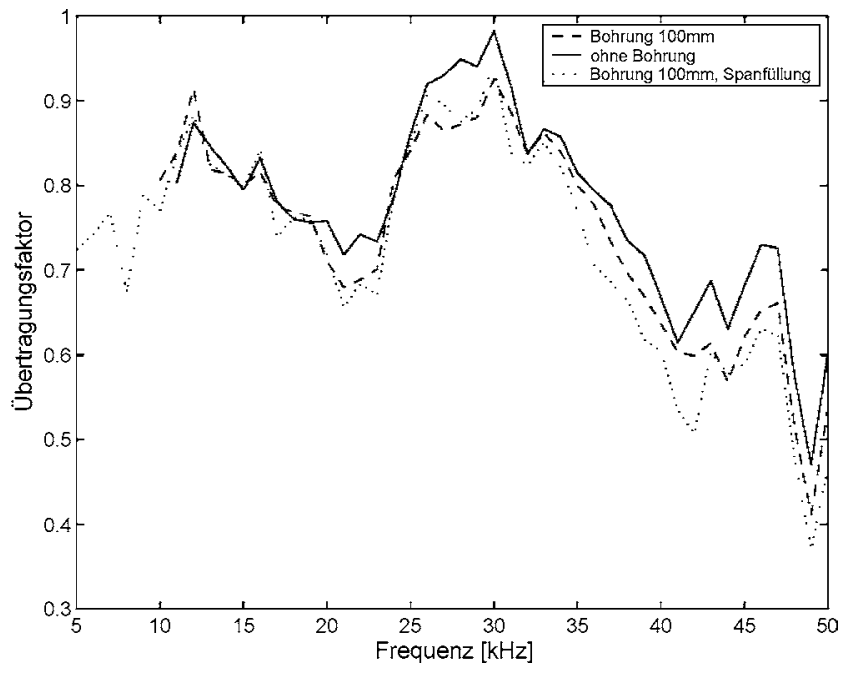

Abb. 4 Frequenzkennlinien des Übertragungsfaktors

Fig. 4 Characteristic lines of frequencies for transfer factor

ren. Es zeigt sich, dass unmanipulierte Prüfkörper das beste Übertragungsverhalten aufweisen. Im Frequenzbereich von $33 \mathrm{kHz}$ bis $49 \mathrm{kHz}$ zeigt sich eine Abnahme des Übertragungsfaktors, wobei bei $46 \mathrm{kHz}$ ein weiteres lokales Maximum vorzufinden ist. Das Minimum im gesamten Frequenzbereich liegt bei allen Kennlinien bei $49 \mathrm{kHz}$. Bei dieser Frequenz ist das Übertragungsverhalten am ungünstigsten. Im Bereich von $25 \mathrm{kHz}$ bis $35 \mathrm{kHz}$ ist das Übertragungsverhalten am besten und die unterschiedlich manipulierten Prüfkörper lassen sich am deutlichsten unterscheiden. Hobelspäne als Füllstoff zeigen keinen Einfluss auf das Übertragungsverhalten.

\subsection{Schallgeschwindigkeit}

Die Messungen zeigen im Mittelwert über den gesamten Frequenzbereich die erwartete Tendenz, dass mit steigender Messfrequenz auch die Schallgeschwindigkeiten größer werden. Alle Prüfkörper zeigen den gleichen Kurvenverlauf (Abb. 5). Bei der Frequenz $22 \mathrm{kHz}$ stellt sich bei allen Messreihen ein lokales Maximum ein.

Die Messreihe ohne Bohrung weist immer die höchsten Schallgeschwindigkeiten auf, deutlich von dieser Kurve getrennt verlaufen die Messwerte der Messreihe mit $30 \mathrm{~mm}$ Bohrung sowie jener mit $100 \mathrm{~mm}$ Bohrung. Die einzelnen Messreihen können je nach Messfrequenz unterschiedlich genau voneinander getrennt werden. In Abb. 6 sind die Quartile der Werteverteilung der einzelnen Gruppen bei der Frequenz $28 \mathrm{kHz}$ aufgezeichnet. Bei dieser Frequenz lassen sich Differenzierungen am günstigsten vornehmen. Anhand der Schallgeschwindigkeiten kann bei den Messreihen mit einer $100 \mathrm{~mm}$ Bohrung nicht unterschieden werden, ob sich Luft, Holzspäne oder Holzstaub darin befinden. 


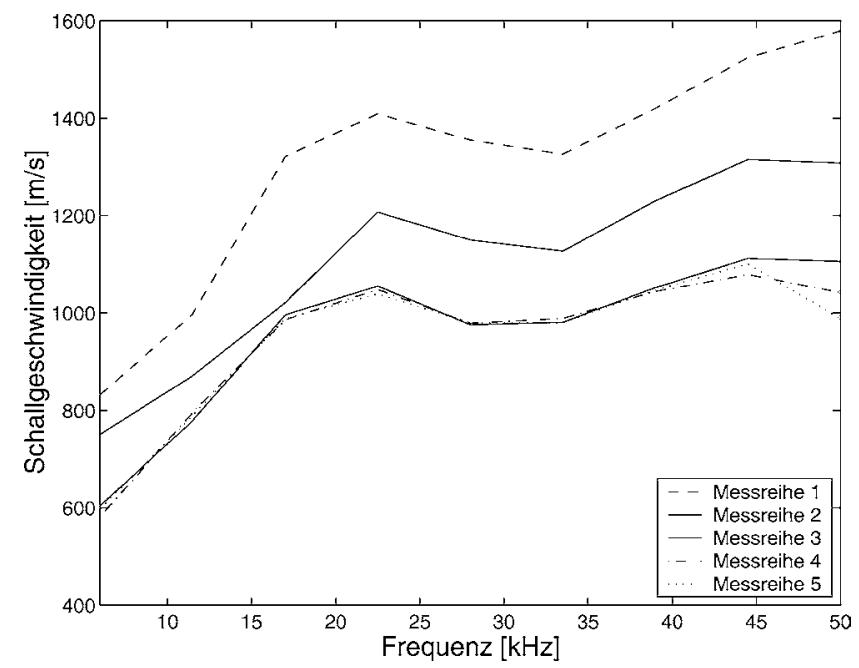

Abb. 5 Schallgeschwindigkeit $(c)$ bei verschiedenen Frequenzen $(f)$. Messreihen 1: ohne Bohrung 2: zentrische Bohrung mit $30 \mathrm{~mm}$ Durchmesser 3: zentrische Bohrung mit $100 \mathrm{~mm}$ Durchmesser 4: zentrische Bohrung mit $100 \mathrm{~mm}$ Durchmesser, mit Spänen aufgefüllt 5: zentrische Bohrung mit $100 \mathrm{~mm}$ Durchmesser, mit Schleifstaub aufgefüllt

Fig. 5 Sound velocities $(c)$ for different frequencies $(f)$. Test series 1: without hole 2: with a hole of $30 \mathrm{~mm}$ diameter 3: with a hole of $100 \mathrm{~mm}$ of diameter 4 : with a hole of $100 \mathrm{~mm}$ of diameter and filled with strands 5: with a hole of $100 \mathrm{~mm}$ of diameter and filled with sanding dust

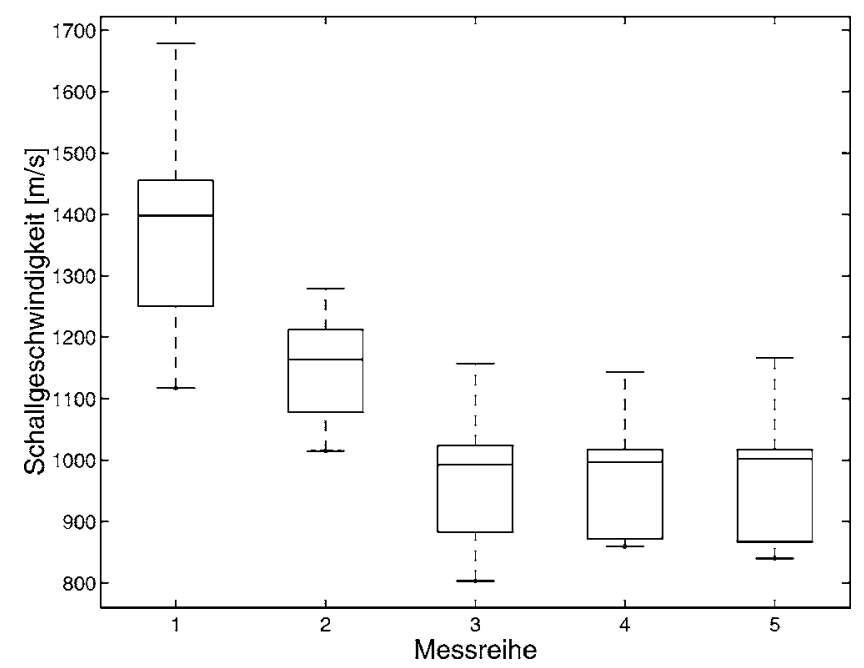

Abb. 6 Schallgeschwindigkeit (c) bei $28 \mathrm{kHz}$ Messfrequenz. Messreihen s. Abb. 5

Fig. 6 Sound velocity (c) at $28 \mathrm{kHz}$. Test series see Fig. 5

\subsection{Dämpfung}

Auch hier ergeben die Mittelwerte der Messungen ähnliche Tendenzen bei zunehmender Messfrequenz (Abb. 7). Bis $40 \mathrm{kHz}$ setzt sich die Messreihe ohne Bohrung deutlich von den anderen ab, gleicht sich aber zwischen 40 und $50 \mathrm{kHz}$ jener mit $30 \mathrm{~mm}$ Bohrung an.

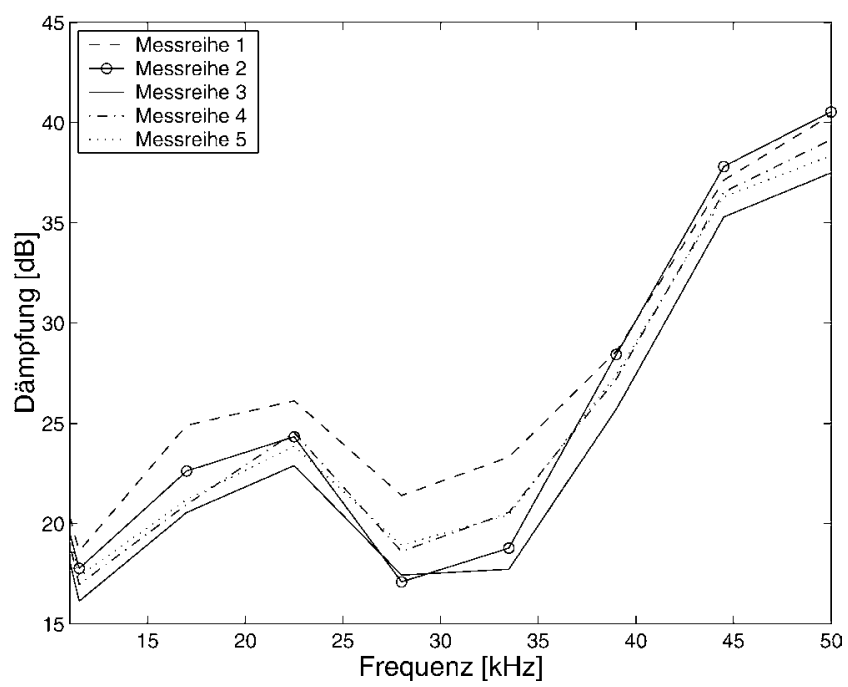

Abb. 7 Dämpfung $(D)$ bei verschiedenen Frequenzen $(f)$. Messreihen s. Abb. 5

Fig. 7 Attenuation $(D)$ for different frequencies $(f)$. Test series see Fig. 5

Eine Betrachtung der Mittelwerte zeigt, dass bei $33,5 \mathrm{kHz}$ die Kurven am besten voneinander unterschieden werden können. Trotzdem können bei keiner der Messfrequenzen die Einzelwerte der Messungen in ähnlich guter Weise in distinkte Gruppen unterschieden werden wie bei der Schallgeschwindigkeit. Es ist festzustellen, dass die größte Dämpfung bei der Messreihe ohne Bohrung und die geringste Dämpfung bei der Messreihe mit $100 \mathrm{~mm}$ Bohrung ohne Füllung auftrat. Die Dämpfungen der Messreihen mit Spanund Staubfüllungen bewegen sich dazwischen. Ein Hohlraum im Inneren des Prüfkörpers begünstigt demnach die Signalübertragung.

\subsection{RMS}

Die ermittelten Effektivwerte der Signale zeigen ebenfalls einen frequenzabhängigen Verlauf (Abb. 8). Daraus ist ersichtlich, bei welcher Frequenz die Signale durch die Prüfkörper besser übertragen werden. Bei $11 \mathrm{kHz}$ ist die Übertragung der Signale der Messreihen 1 und 2 am besten. Die Signale der Messreihen 3 bis 5 werden dagegen bei einer Frequenz von $28 \mathrm{kHz}$ am besten übertragen. Im Bereich von $20 \mathrm{kHz}$ bis $25 \mathrm{kHz}$ sowie bei $6 \mathrm{kHz}$ ist ein starkes Absinken des Effektivwertes zu verzeichnen. Eine Differenzierung der Messreihen anhand des Effektivwertes ist nicht möglich, da die Streuung der Messwerte zu groß ist.

\subsection{Peak to Peak-Wert}

Abbildung 9 zeigt die errechneten Peak to Peak-Werte. Es bildet sich der gleiche frequenzabhängige Verlauf wie bei der Kennline des RMS ab. Es wurden bei der Ermitt- 


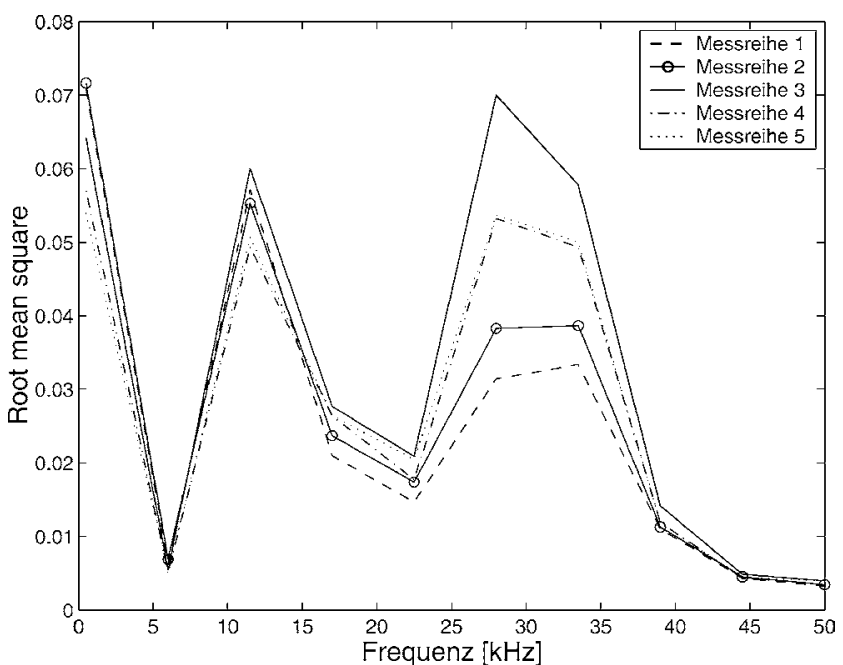

Abb. 8 RMS bei verschiedenen Frequenzen $(f)$. Messreihen s. Abb. 5

Fig. 8 RMS for different frequencies $(f)$. Test series see Fig. 5

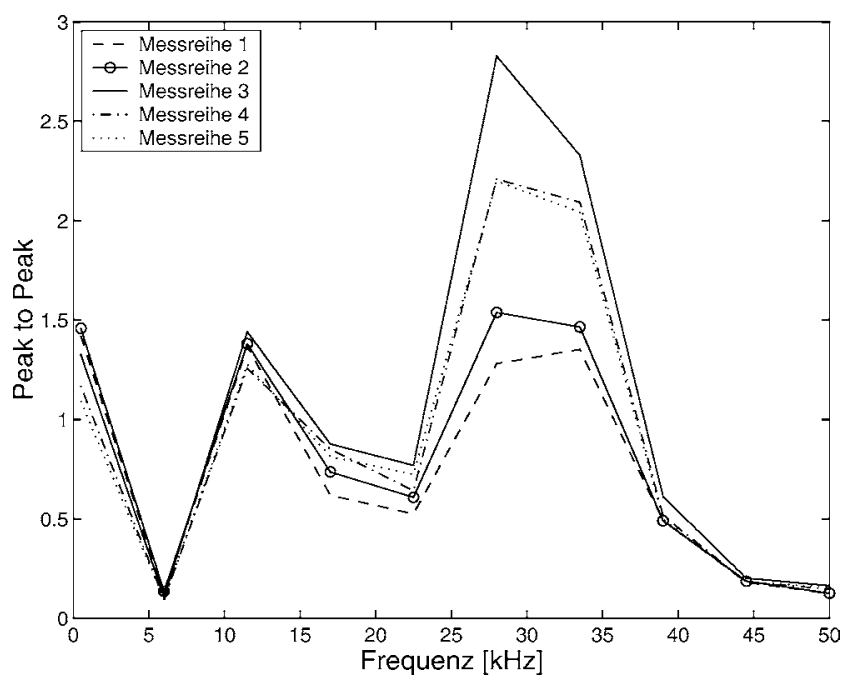

Abb. 9 Peak to Peak bei verschiedenen Frequenzen $(f)$. Messreihen s. Abb. 5

Fig. 9 Peak to Peak for different frequencies $(f)$. Test series see Fig. 5

lung des Peak to Peak-Wertes große Streuungen innerhalb der Messreihen festgestellt, sodass eine Differenzierung der Messreihen anhand dieses Kennwertes nicht möglich ist.

\subsection{Signalenergie}

Aus Abb. 10 wird deutlich, dass in einem Frequenzbereich von $22 \mathrm{kHz}$ bis $40 \mathrm{kHz}$ bei den Messreihen mit einer Bohrung von $100 \mathrm{~mm}$, sowohl mit als auch ohne Füllung, deutlich mehr Energie übertragen wird als bei den übrigen Messreihen. Weiterhin ist zu bemerken, dass bei einer Frequenz von $28 \mathrm{kHz}$ bei den Messreihen 3, 4 und 5 die meiste Energie übertragen wird. Bei den Messreihen 1 und 2 wird

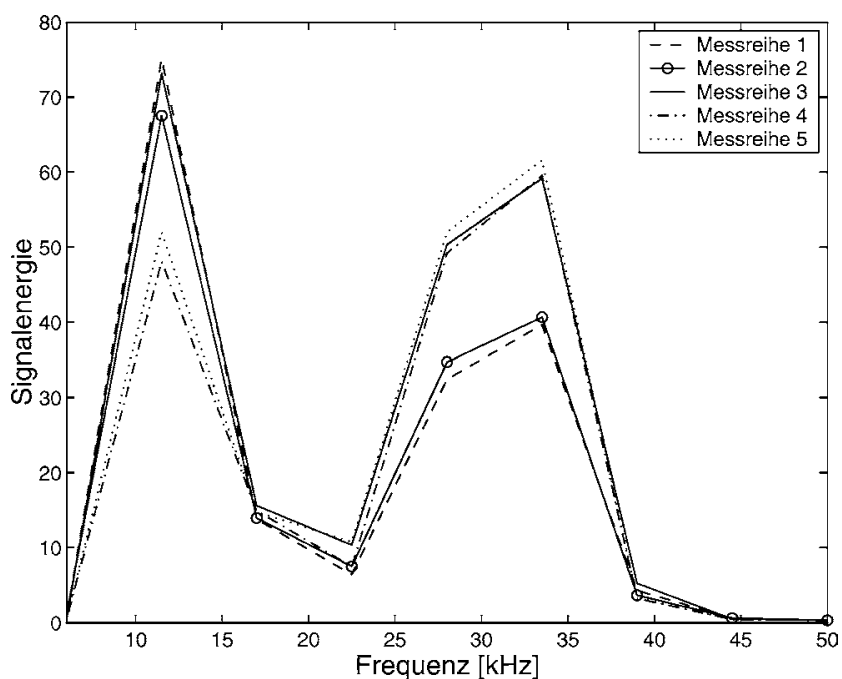

Abb. 10 Signalenergie bei verschiedenen Frequenzen $(f)$. Messreihen s. Abb. 5

Fig. 10 Signal energy for different frequencies $(f)$. Test series see Fig. 5

die meiste Energie dagegen bei $11,5 \mathrm{kHz}$ übertragen. Diese Aussagen lassen sich jedoch statistisch nicht belegen, da sich die Streuung der Werte als zu groß erweist. Auch aus den Absolutwerten bei einer beliebigen Frequenz lassen sich keine sicheren Erkenntnisse ziehen.

\section{Zusammenfassung}

Es wurden verschiedene Kennwerte herangezogen, um die Übertragung eines Ultraschallsignals durch einen Stamm $\mathrm{zu}$ charakterisieren. Aus den Ergebnissen wird ersichtlich, dass sich die Schallgeschwindigkeit am besten eignet, um die Fehlstellen in den Prüfkörpern zu charakterisieren. Mithilfe der Schallgeschwindigkeit lassen sich die Prüfkörper ohne Bohrung sowie mit einer Bohrung von $30 \mathrm{~mm}$ Durchmesser deutlich von den Prüfkörpern mit einer Bohrung von $100 \mathrm{~mm}$ Durchmesser unterscheiden. Eine Differenzierung der Prüfkörper, deren Hohlraum mit Spänen oder Staub gefüllt wurde, ist nicht möglich. Diese Dichteunterschiede haben keinen Einfluss auf die Schallgeschwindigkeit. Als Grund ist hierfür anzusehen, dass sich die Welle schneller um den Kernbereich herum fortpflanzt, egal ob ein Füllstoff vorhanden ist oder nicht. Durch die Untersuchung konnte eine Frequenzabhängigkeit der Schallgeschwindigkeit bestätigt werden. Als weitere Kenngröße wurde die Dämpfung des Signals einbezogen. Die Dämpfung der Prüfkörper ohne Bohrung ist im Mittel zwar deutlich höher als bei den übrigen Prüfkörpern, jedoch lässt die Streuung der Messwerte keine eindeutige Zuordnung der Prüfkörper zu. Wie die Schallgeschwindigkeit zeigt auch die Dämpfung einen frequenzabhängigen 
Verlauf. Bei $11 \mathrm{kHz}$ sowie zwischen $28 \mathrm{kHz}$ und $33 \mathrm{kHz}$ ist die Dämpfung der Signale am geringsten. Die Kennwerte RMS, Peak to Peak sowie die Signalenergie liefern Ergebnisse, die keine Differenzierung der Prüfkörper zulassen. Alle Kennwerte außer der Schallgeschwindigkeit sind von der Signalamplitude abhängig. Daraus kann geschlussfolgert werden, dass diese sehr stark von anderen Einflüssen wie Holzfeuchte oder Dichte sowie den Ankopplungsbedingungen beeinflusst wird. Diese Einflüsse lassen sich jedoch messtechnisch nicht ausreichend von den zu prüfenden Größen trennen. In der praktischen Anwendung kann somit einzig auf die Schallgeschwindigkeit zur Beurteilung von Fehlstellen in Baumstämmen zurückgegriffen werden.

Danksagung Die Arbeiten erfolgten im Rahmen einer Belegarbeit von Herrn Clauss an der ETH Zürich. Sie sind Teil eines von der Velux-Stiftung, Zürich geförderten Projektes

\section{Literatur}

Bethge K, Mattheck K, Hunger E (1996) Geräte zum Auffinden und Bewerten von holzzerstörender Fäule in Bäumen. Vortrag zum 2. VTA-Spezialseminar „Messen und Beurteilen am Baum“, Karlsruhe, 8.-9.5.1996
Bucur V (1995) Acoustics of Wood. CRC Press, Boca Raton New York London Tokyo

Dokumentation Messsystem USH (2003) Fraunhofer-Institut für zerstörungsfreie Prüfverfahren

Frankenstein B, Augustin J, Neunübel H (2003) Ergebnisbericht USBewertung Holz, Einrichtung für Akustische Diagnose und Qualitätssicherung

Große CU, Reinhardt HW (1999) Schallemissionsquellen automatisch lokalisieren. Materialprüfung 41:342-347

Kurz JH, Große CU, Reinhardt HW (2004) Strategies for reliable automatic onset time picking of acoustic emissions and of ultrasound signals in concrete. Ultrasonics 43:538-546

Mattheck C, Bethge K (1992) Ein Impulshammer mit Laufzeitmessung zum Auffinden von Faulstellen in Holz. Kernforschungszentrum Karlsruhe

Niemz P, Bächle F (2002) Bohrwiderstands- und Schallgeschwindigkeitsmessung Untersuchungen zur Erkennbarkeit von Fäule in Fichtenholz. Stadt Grün 19:52-55

Niemz P, Bues CT, Herrmann S (2002) Die Eignung von Schallgeschwindigkeit und Bohrwiderstand zur Beurteilung von simulierten Defekten in Fichtenholz. Schweiz Z Forstwes 6(153): 201-209

Niemz P, Kucera L (1997) Einfluss künstlich erzeugter Defekte und natürlicher Holzfehler auf die Schallausbreitungsgeschwindigkeit. Holz-Zentralblatt 123(50):7-96

Sandoz JL, Lorin P (1994) Evaluation de l'état physico-mechanique de l'arbre sur pied par ultrason, Protocole de mesures, Lausanne Informationsschrift IBOIS

Schwarze F, Fink S (1994) Die Ermittlung der Holzzersetzungsmuster am lebenden Baum. Neue Landsch 39:182-193 